\title{
Analysis of the experimental parameters impacting Non-Photochemical Laser-Induced nucleation of glycine in water
}

\author{
N. Bosnjaković-Pavlović ${ }^{1}$, B. Clair ${ }^{1}$, A. Ikni ${ }^{1}$, W. Li' ${ }^{2}$, S. Novaković ${ }^{3}$, P. Gemeiner ${ }^{1}$, P. Scouflaire ${ }^{4}$, \\ A. Spasojević-de Biré ${ }^{1 *}$ \\ ${ }^{1}$ Laboratory "Structures Propriétés et Modélisation des Solides" (SPMS), CNRS, UMR 8580, Université Paris-Saclay, \\ CentraleSupélec, 8-10 rue Joliot-Curie,91190 Gif-sur-Yvette, France \\ ${ }^{2}$ BUCT Paris Curie Engineer School, Beijing, China \\ ${ }^{3}$ Laboratory "Énergétique Moléculaire et Macroscopique, Combustion" (EM2C), CNRS, UPR 288, Université Paris-Saclay, \\ CentraleSupélec, 8-10 rue Joliot-Curie,91190 Gif-sur-Yvette, France \\ ${ }^{4}$ Department of Physical Chemistry, Vinča Institute of Nuclear Sciences - National Institute of the Republic of Serbia, University of \\ Belgrade, Serbia \\ anne.spasojevic@centralesupelec.fr
}

Non-Photochemical Laser-Induced nucleation (NPLIN) is a promising nucleation technique [1] for which more than eighty papers have been published. In an NPLIN experiment, a supersaturated solution of a molecule is irradiated by a laser (pulsed or continuous, focused or nonfocused) that induces the molecule's nucleation. Even though glycine nucleation constitutes almost one-quarter of these research activities reported in the literature, the impact of different experimental conditions on its nucleation is still not fully understood [2]. NPLIN of glycine in water has been demonstrated at different molarities and different energy densities induced using a non-focalized pulsed laser $(532 \mathrm{~nm})$ at $290 \mathrm{~K}$. A new index (Ind50), allowing easy comparison with the literature, was used to characterize the impact of molarities and energy densities on the nucleation efficiency. A threshold index (IndThrs ( )) indicating the minimum energy density required to obtain in a given experimental condition one crystal per vials in average has been determined. The impact of the circular or linear polarization of the laser beam on the glycine polymorphism ( - or -glycine) has been studied and characterized using a third new index named NPLIN determinant. The experimental interface (glass-solution or air-solution) gives the opposite polymorphism behavior. The relationship between devices, solution, and experimental conditions and observable such as nucleation efficiency, nucleation site, induction time, crystal counting, and polymorphism have been modelized in a mind-map (figure

1). Within this context, this work is a contribution towards a better understanding of the impact of experimental conditions on NPLIN nucleation that will permit a better design and control of NPLIN experimental setups.

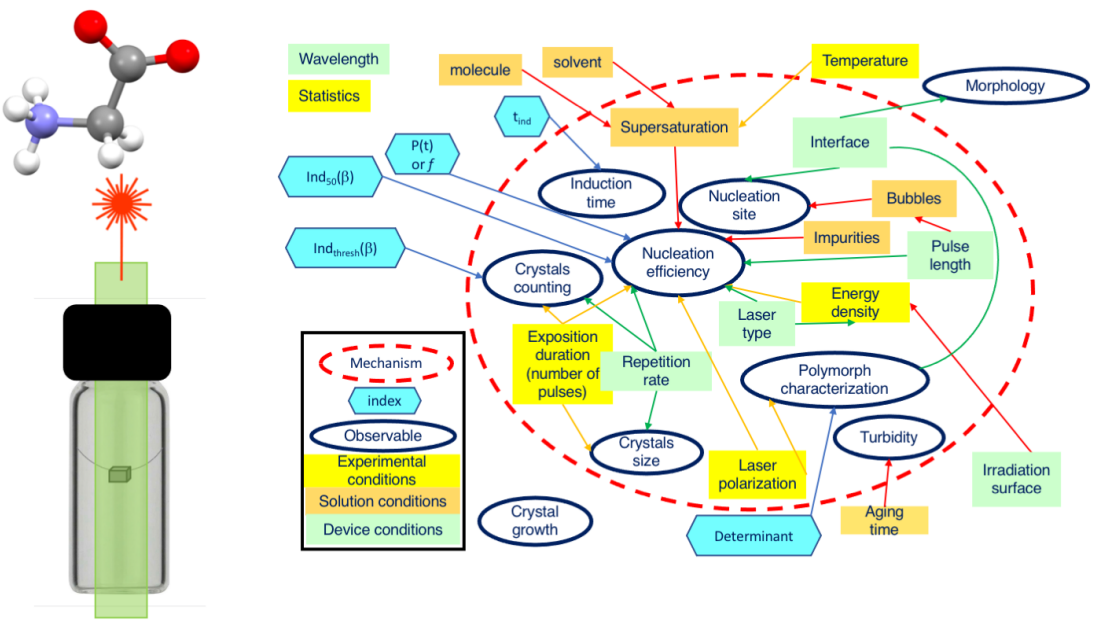

Figure 1. NPLIN mind-map parameters. Parameters inside the red dashed circles has a significant influence on the mechanism.

[1] Garetz, B. A.; Aber, J. E.; Goddard, N. L.; Young, R. G.; Myerson, A. S. (1996) Phys. Rev. Lett. 77, 3475-3476.

[2] Clair, B.; Ikni, A.; Li, W.; Scouflaire, P.; Quemener, V.; Spasojević-de Biré, A. (2014) J. Appl. Crystallogr., 47, 1252-1260

Keywords: laser-induced nucleation; NPLIN; glycine; polymorphism; polarization switching 